\title{
Neurofeedback for treating tinnitus
}

\author{
Katalin Dohrmann ${ }^{1, *}$, Nathan Weisz ${ }^{2}$, Winfried Schlee ${ }^{1}$, Thomas Hartmann ${ }^{1}$ and \\ Thomas Elbert ${ }^{1}$
}

\author{
${ }^{1}$ University of Konstanz, Department of Psychology, Box D 25, 78457 Konstanz, Germany \\ ${ }^{2}$ INSERM - Unite 821, Centre Hospitalier Le Vinatier, Batiment 452, 69500 Bron, France
}

\begin{abstract}
Many individuals with tinnitus have abnormal oscillatory brain activity. Led by this finding, we have developed a way to normalize such pathological activity by neurofeedback techniques (Weisz et al. (2005). PLoS Med., 2: e153). This is achieved mainly through enhancement of tau activity, i.e., oscillatory activity produced in perisylvian regions within the alpha frequency range $(8-12 \mathrm{~Hz})$ and concomitant reduction in delta power range $(0.5-4 \mathrm{~Hz})$. This activity is recorded from electrodes placed on the frontal scalp. We have found that modification of the tau-to-delta ratio significantly reduces tinnitus intensity. Participants who successfully modified their oscillatory pattern profited from the treatment to the extent that the tinnitus sensation became completely abolished. Overall, this neurofeedback training was significantly superiorin reducing tinnitus-related distress than frequency discrimination training.
\end{abstract}

Keywords: neurofeedback; EEG; tinnitus

\section{Tinnitus in relation to abnormal oscillatory brain activity?}

Subjective tinnitus, an auditory sensation that appears without the presence of an external physical source of the sound is a symptom rather than a nosological entity. Treatments of this condition have included cognitive-behavioral therapy (Andersson, 2002), psychotherapy (Goebel, 2001), "tinnitus retraining therapy" (Jastreboff and Jastreboff, 2006), biofeedback (Landis and Landis, 1992), physical therapy (Rief et al., 2005), transcranial magnetic stimulation (Langguth et al., 2006), auditory frequency training (Flor et al., 2004), prolonged rest and relaxation (Weber

\footnotetext{
*Corresponding author. Tel.: + $33(0) 472138916$; Fax: + 49753188 4601;

E-mail: katalin.dohrmann@uni-konstanz.de
}

et al., 2002), and the administration of such agents as lidocaine (Kalcioglu et al., 2005). Despite isolated reports of success, no treatment has been found to be effective in general (Dobie, 1999).

Several central nervous phenomena have been proposed to explain the emergence of tinnitus. However different, all assume a decisive role of reduced afferent input to central auditory regions usually due to damaged hair cells of the cochlea. One proposed mechanism draws analogies to somatosensory phantom pain, where somatotopic maps reorganize as a result of altered afferent input (Elbert and Heim, 2001). Reduced sensory input to central neurons may change their receptive fields and make them become sensitive to input from neighboring regions through expression of neural plasticity (see Chapter 3). In the auditory system this may lead to an overrepresentation of audiometric edge frequency regions, and that may 
cause the phantom sensation of tinnitus. In the somatosensory system such map reorganization, has been shown to be linearly correlated with the amount of phantom limb pain (Flor et al., 1995) (Chapter 4). Reorganization of the auditory cortex occurs after hearing loss as shown in many studies in animals (Eggermont and Roberts, 2004). One magnetoencephalographic (MEG) study in humans with tinnitus has shown distortions of the tonotopic map in auditory cortex that was related to the tinnitus sensation (Muhlnickel et al., 1998).

Training that aims at reestablishing afferent input would be expected to reverse maladaptive reorganization. Clinical studies have used frequency discrimination trainings (FDTs) targeted at the region around the tinnitus frequency (Flor et al., 2004), or within the deafferented frequency region (see Fig. 4, p. 494), but without noticeable benefit regarding tinnitus. Nevertheless, these negative clinical findings need not necessarily contradict that map reorganization may be sufficient to produce tinnitus (Weisz et al., 2006) (Chapter 6). Hair-cell damage that alters the cochlear frequency processing may have interfered with the attempts to direct afferent input to specific neuronal assemblies.

Deprivation of input to the auditory system may alter spontaneous activity in groups of neurons. Given that tinnitus is a continuous sensation, there should be an ongoing neural signal that codes the perpetual sensation. The question one must ask is how does intrinsic activity elicit a sensation? Different assumptions from animal research have been made regarding the relation between altered neural activity and tinnitus. It has been hypothesized that tinnitus may be caused by increase of firing rates or altered synchrony of firing (Eggermont and Roberts, 2004). Since the onset of tinnitus usually follows noise trauma, which also alters neural synchrony (Norena and Eggermont, 2003), these two phenomena may be related. In individuals with tinnitus, we found an abnormal ongoing spontaneous pattern of magnetic brain activity (Weisz et al., 2005). Both MEG and electroencephalography (EEG) represent the summed activity of many neurons and reflect synchronous activity in entire neuronal populations. In individuals with tinnitus abnormalities in the MEG are pronounced in recordings from temporal regions where slow waves in the delta range $(0.5-4 \mathrm{~Hz})$ are increased, and the alpha activity $(8-12 \mathrm{~Hz})$ is reduced. The abnormalities were strongly correlated with the level of tinnitusrelated distress (see Fig. 1).

Similar patterns were observed in other disorders, and Llinas et al. (2005) hypothesized that deprivation of input in general (in this case hearing loss), leads to hyperpolarized thalamic neurons, which in turn trigger bursting activity at $\sim 4 \mathrm{~Hz}$ (Jeanmonod et al., 1996). The bursting activity spreads across large parts of the cortex and can be detected on a macroscopic level as changes in recorded MEG or EEG. The slow-wave activity in $\mathrm{MEG}$ or EEG recordings could also be the result of hypoactivation of cortical neurons. The described bottom-up process could also activate top-down pathways by input from cortico-limbic pathways, which modulate emotional and motivational processing. Slow oscillatory activity could be the neural signature of the coupling between anatomically distant brain regions, as occurs in normal information processing (Sauseng et al., 2005). However, enhanced slow oscillatory activity was not the only observed abnormality in the MEG of individuals with tinnitus; reduction of alpha power around $10 \mathrm{~Hz}$ in recordings from temporal regions (so-called tau rhythms) is hypothesized to be a normal reaction to sound (Hari and Salmelin, 1997; Lehtela et al., 1997). As individuals with tinnitus continuously hear an internal sound, it is conceivable that the power of their tau rhythms is reduced. Weisz et al. 2007 (Chapter 6) provide more information regarding the relationship between ongoing oscillatory activity and tinnitus.

Slow wave EEG activity, especially that occurring during sleep, has been associated with brain silence and the decrease of thalamic input to the cortex (Steriade and Timofeev, 2003). In the waking state, slow waves in the EEG occur in many developmental and degenerative disorders, in toxic and metabolic encephalopathy, and in other neurological conditions. The anatomical location of focal neural generators of slow waves is usually in the vicinity of structural lesions such as cerebral 

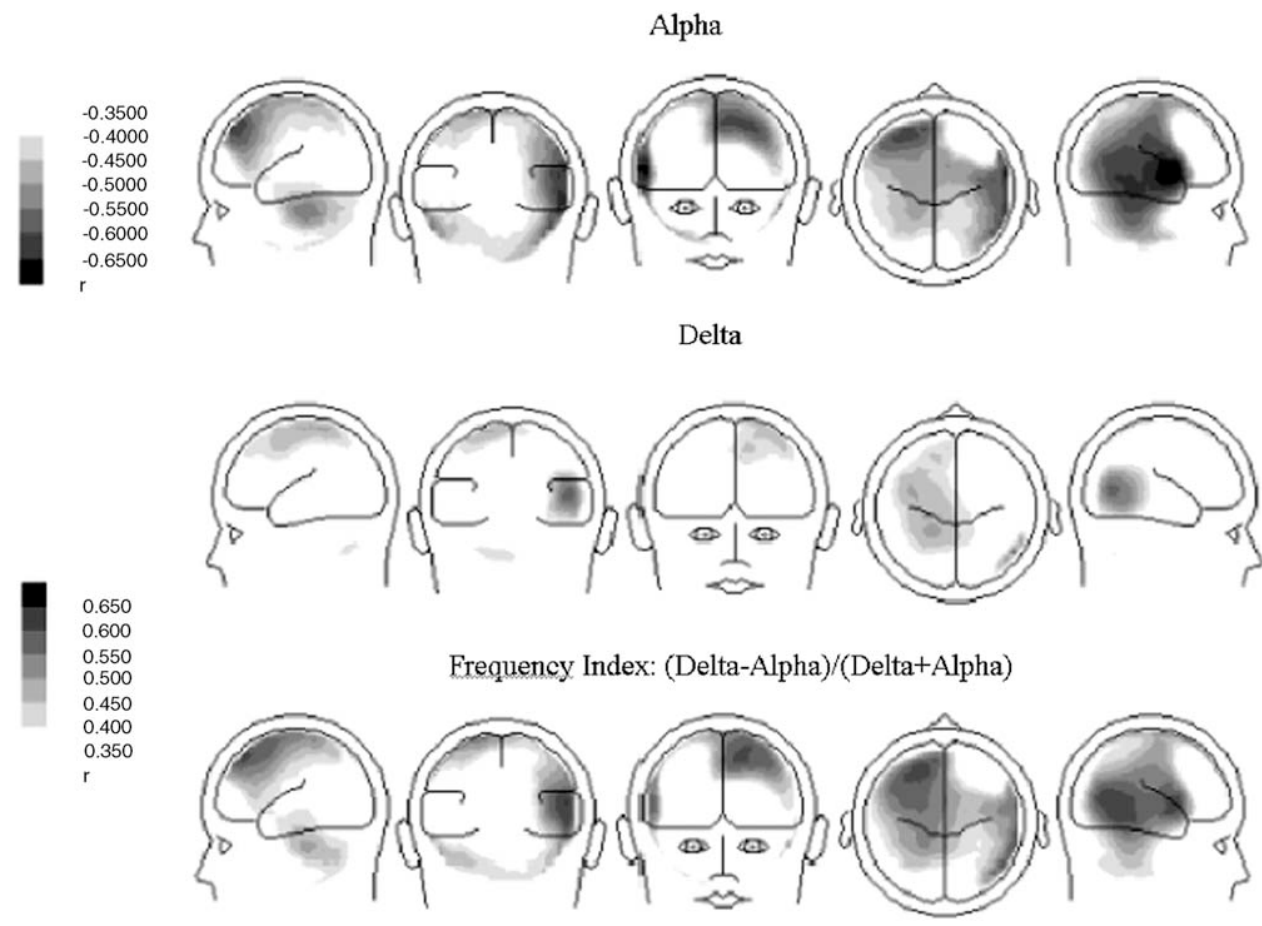

Fig. 1. Distribution of correlation coefficients between the tinnitus-related distress (score on the Tinnitus Questionnaire) and the power in alpha, delta, and a frequency index of both bands, respectively. Effects are largest for right temporal and left frontal sources. (Adapted with permission from Weisz et al., 2005.)

infarcts, contusions, local inflammations, tumors, and subdural hematoma (Walter, 1936; Tanaka et al., 1998; de Jongh et al., 2003). The output of these generators varies with the level of metabolism and blood flow and depends on the magnitude of the insult (Strik et al., 2002; Hensel et al., 2004).

We consequently propose that neural networks in at least three regions are involved in tinnitus, namely: temporal areas, which are relevant for the processing of perceptual features of tinnitus, and frontal and limbic regions, which are involved in processing the emotional and motivational features (i.e., the perceived distress) of the phantom sound. Slow oscillatory activity couple the involved regions together. The normal auditory alpha/tau rhythms are reduced due to a permanent processing of this internal sound (tinnitus). If these elements were of fundamental importance in the generation of tinnitus, normalization of the aberrant rhythms would be expected to alleviate tinnitus. In the present study, we have explored the possibility to modify this abnormal activity through the technique of neurofeedback, i.e., by recording the EEG signal, extracting relevant parameters, and displaying this information to the person with tinnitus.

\section{Neurofeedback: background and previous application}

In individuals with chronic tinnitus the aim was to modify the aberrant rhythms in the EEG mainly the enhanced delta and reduced tau power. The technique is described in detail elsewhere (Dohrmann et al., submitted). If the abnormal brain rhythms were causally related to the tinnitus, it would be expected that changing the spontaneous activity pattern would modify the tinnitus and its associated distress.

Neurofeedback has proven to be effective in modifying the characteristics of spontaneous and 
evoked brain activity (see review by Rockstroh et al., 1984). By means of an EEG-based training method, individuals can learn to self-regulate distinct features of their ongoing brain activity, such as the power in a certain frequency band.

The principles of neurofeedback are: the EEG signal is recorded, processed, and converted into a contingent auditory and/or visual feedback signal for the patient. Training for the task of neurofeedback aims at teaching the patient an association between the signal and the most recent mental state. Instantaneous feedback to the patient is crucial for training. If the patient is successful in changing the EEG and the brain activity reaches a certain pre-defined level, the patient receives a reward, such as a smile or applause.

Research using neurofeedback began in the late 1960s with work on uncontrolled epilepsy as well as training in alpha feedback for relaxation (Nowlis and Kamiya, 1970; Travis et al., 1974). Today, it is applied to the field of epilepsy (Elbert et al., 1991; Rockstroh et al., 1993) and attention deficit/ hyperactivity disorders (AD/HD) (e.g., Monastra et al., 2000; see also Rockstroh et al., 1990). Although the investigation of neurofeedback in treatment of $\mathrm{AD} / \mathrm{HD}$ stands in the forefront, there is a lack of standardized parameters of implementation (such as number of sessions, controlled clinical trials, parameters of success, registration of the EEG activity; see Duffy, 2000; Ramirez et al., 2001).

Studies exploring the effect of neurofeedback on tinnitus are scarce. Two studies (Gosepath et al., 2001; Schenk et al., 2005) have supported the assumption that distress in general is associated with a reduction in the power in the alpha band recorded from the posterior scalp and enhancement of the power in the beta $(14-30 \mathrm{~Hz})$ band. On the basis of these findings it has been hypothesized that the vicious circle of strain, anxiety, and depression that is initiated in tinnitus can be interrupted through relaxation and by upregulating the alpha activity (sign of increased relaxation) and downregulating the beta activity (sign of decreased stress).

The approach described in this chapter differs essentially in that the activity being modified is different in terms of its anatomical localization and its presumed type of generators (Dohrmann et al., submitted). While posterior sites have been the regions of interest in many studies, we focus on temporal and frontal regions, which we believe are mainly involved in the psychoacoustic and distress aspects of chronic tinnitus. We therefore record EEG from $\mathrm{F} 3, \mathrm{~F} 4, \mathrm{Fc} 1$, and $\mathrm{Fc} 2$ positions on the scalp. First, the tau activity is in the frequency range of alpha activity but is presumed to be generated in sylvian regions of the brain, including the auditory cortex, from where it projects to frontal regions. Secondly, the slow-wave activity would be an indication of deafferented tinnitusrelated neuronal networks and not only a sign of general distress. More specifically, the deafferented thalamus might degenerate causing the respective cortical drive to cease.

While it seems logical to assume that distress from tinnitus is related to its loudness it is also conceivable that a relief of distress results in a reduction of the tinnitus intensity. Nevertheless, it has been reported that loudness and distress are relatively marginally connected (Henry and Meikle, 2000). These conclusions could, however, depend on the procedures used to treat tinnitus. Most therapies focus on coping with the annoyance of the tinnitus and less on reduction or abolishment of the tinnitus itself (Andersson and Lyttkens, 1999). The advantage of neurofeedback over input-based (sound) therapies such as FDT, which are based on assumptions of central nervous reorganization, is that the treatment using neurofeedback is not affected by hearing loss.

\section{Effects of a specific tau/delta neurofeedback training}

Based on the hypotheses outlined above, we compared the effectiveness of different neurofeedback protocols in reducing the tinnitus in 21 individuals with chronic tinnitus ( 9 females and 12 males, age $31-62$, median 48 years). The mean duration of their tinnitus was 8.7 years $(\mathrm{SD} \pm 7.4)$, the mean tinnitus intensity of $25 \mathrm{~dB}$ HL ( $\mathrm{SD} \pm 11.7)$, and a mean distress level of 26.5 points (slight distress; $\mathrm{SD} \pm 15.3$ ) on the Tinnitus Questionnaire (Goebel 


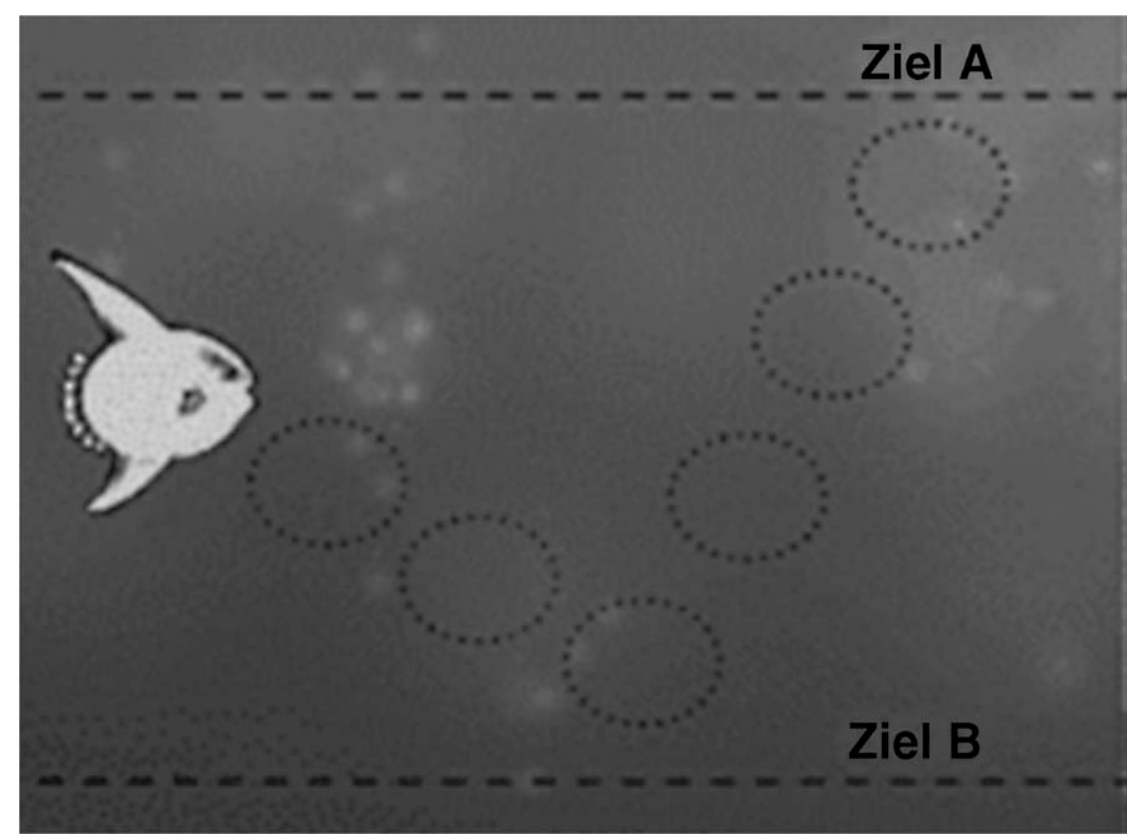

Fig. 2. The schematic fish on the patient monitor that was to be "moved" up or down (depending on the feedback protocol) during neurofeedback training. The height of the fish represented the amplitude/power of the specific frequency band. (By courtesy of Eldith $\mathbb{C}$ Corporation, Germany.) (See Color Plate 46.2 in color plate section.)

and Hiller, 1998), distress scale from 0 (no distress) to 84 points (very severe distress).

The participants in the study underwent a training program comprising 10 sessions (net training time per session: $30 \mathrm{~min}$ ) over the course of 4 weeks. EEG was recorded from electrodes place at fronto-central positions ( $\mathrm{F} 3, \mathrm{~F} 4, \mathrm{Fc} 1$, and $\mathrm{Fc} 2)$. Eleven participants aimed at enhancing the ratio of tau-to-delta power (TDR), five participants aimed at enhancing tau power (without feedback about delta power), and five participants aimed at reducing delta power (without feedback about tau power).

Feedback was provided through the use of a symbol (e.g., a fish; see Fig. 2) that moved from left to right on a computer screen. A rise of the symbol indicated successful enhancement of the TDR and the tau power, respectively. In the delta protocol group, a drop in the symbol indicated successful decrease of delta power. A sunshine symbol indicated that the participant had reached an individually adjusted threshold. Participants were given no particular instructions on how to obtain the anticipated change in the EEG activity but they should change their mental activity to get the symbols on the screen to move in the anticipated way. We also asked them not to engage in any muscular activity, including eye movements and blinks that they might think would facilitate the changes in their EEG activity as indicated by the movement of the figures on the screen.

We monitored training success by matching the intensity of the tinnitus to a $1 \mathrm{kHz}$ test tone (psychoacoustic measure using the audiometer) as well as by measuring the power in the appropriate frequency bands of the resting EEG each before and after the training. It must be emphasized that the participants in the study were unaware of the intensity values they matched to their tinnitus. Various measures of distress related to tinnitus were surveyed once a week using a German Tinnitus Questionnaire (Goebel and Hiller, 1998). Tinnitus status was also measured at a 6-week and 6-month follow-up post-training. 


\section{EEG normalization}

For every pre and post session we calculated the tau-to-delta power ratio and the results showed a significant enhancement of this TDR with an effect size of 0.67. Overall, participants showed an improvement of $71 \%$ (ranging from $32 \%$ to $325 \%$ ). As shown in Fig. 3, participants improved with regard to normalization of their TDR within a session (after training the EEG power is above its values before the training). This normalization develops gradually between sessions, confirmed by an ascending linear trend for pre $(p=0.016)$ and post $(p=0.007)$ TDR-values.

The achieved normalization of the EEG did not differ significantly among groups with different feedback protocols $(p=0.8$, repeated measures ANOVA with between-subject factor "group" comprising the three levels: tau, delta, and tau/ delta) (Table 1).

Many of the participants who were extraordinarily successful in controlling their brain oscillation reported that they would start a session by putting themselves in a mental state of relaxation using approaches they had learned in courses on autogenic training or that they developed personally. One participant who achieved complete relief from tinnitus at the end of treatment had imagined good experiences of job-related success.

\section{Reduction of tinnitus}

Starting with a tinnitus intensity of $25 \mathrm{~dB}$ HL on average $(\mathrm{SD} \pm 11.7)$, participants reduced the intensity of their tinnitus to a mean of $16 \mathrm{~dB}$ HL $(\mathrm{SD} \pm 13.3)$ after the training period and then maintained the intensity level below baseline for 6 weeks and 6 months later $(20 \pm 13.7 \mathrm{~dB}$ and $17.4 \pm 11.9 \mathrm{~dB}$, respectively). During training, the intensity of the tinnitus did not decrease as

Table 1. Allocation of the three different neurofeedback protocols

\begin{tabular}{|c|c|c|c|}
\hline Group & $\begin{array}{l}\text { Group } \\
\text { size }\end{array}$ & $\begin{array}{l}\text { Feedback } \\
\text { measure }\end{array}$ & Treatment goal \\
\hline 1 & 5 & Tau $(8-12 \mathrm{~Hz})$ & Enhancement of tau \\
\hline 2 & 5 & Delta $(1-4 \mathrm{~Hz})$ & Reduction of delta \\
\hline 3 & 11 & Tau/delta-ratio & $\begin{array}{l}\text { Enhancement of the } \\
\text { tau/delta-ratio }\end{array}$ \\
\hline
\end{tabular}

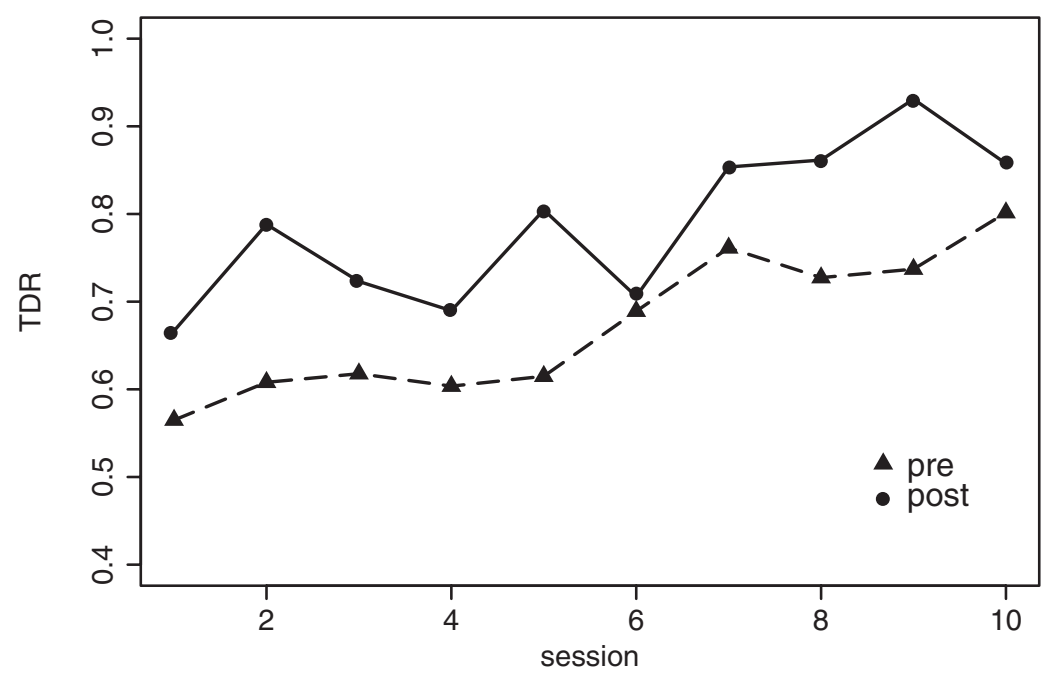

Fig. 3. Development of the mean tau/delta values in the 5 min resting EEG before (dashed line and triangles) and after (filled circles and solid lines) the neurofeedback session. The values are calculated over the whole training group $(n=21)$, independent of the three feedback protocols. It is evident that the post-tau/delta ratio of every session lies above the pre-ratio (within learning effect; even the differences are not significant, tested with $t$-test). Moreover, there is an improvement between the sessions, showing a gradually ascending linear trend. 
gradually as the EEG power ratio, but did so substantially in the first five sessions, and to a lesser extent in the second half of the training. Again, there is no significant difference between the reductions of the three protocol groups.

The individuals who participated in the training reduced their tinnitus-related distress from a mean of $27(\mathrm{SD} \pm 15.3)$ points on the Tinnitus Questionnaire before training to a mean of 19 points $(\mathrm{SD} \pm 15.3)$ after the tenth session. In the followup measures participants showed a mean of 20 points $(\mathrm{SD} \pm 18.0)$ after 6 weeks and a mean of 20.5 points $(S D \pm 20.2)$ after 6 months. It should be noted that changes from the very low baseline level are indeed noteworthy given that many clinical studies only investigate participants presenting with high levels of distress.

\section{Frequency discrimination training}

The outcome of the pooled neurofeedback groups $(N=21)$ was compared to a group $(N=27)$ who received auditory FDT targeted to achieve map normalization, i.e., a reduction of the hearing-loss induced reorganization of neural structures. The use of FDT was inspired by the finding of map reorganization in some individuals with tinnitus (Muhlnickel et al., 1998).

There were 27 participants, 23 men and 4 women, in the FDT group; their age ranged from 24 to 65 years with a median age of 55 . The mean duration of their tinnitus was 9.1 years $(\mathrm{SD} \pm 7.58$, range of 1-32 years).

Both the neurofeedback and the FDT group received 10 training sessions and 1 pre-training session in which they received information about the procedures they were to undergo. The level of distress at baseline is similar for the two groups (neurofeedback: mean of $27(\mathrm{SD} \pm 15.3)$ points; FDT: mean of $34.5(\mathrm{SD} \pm 16.4)$ at first baseline and $29.5(\mathrm{SD} \pm 16.8)$ directly before training start).

Participants in the neurofeedback group had a significant decrease in the distress scores (Fig. 4) while the distress experienced by the participants in the FDT was not significantly affected by the

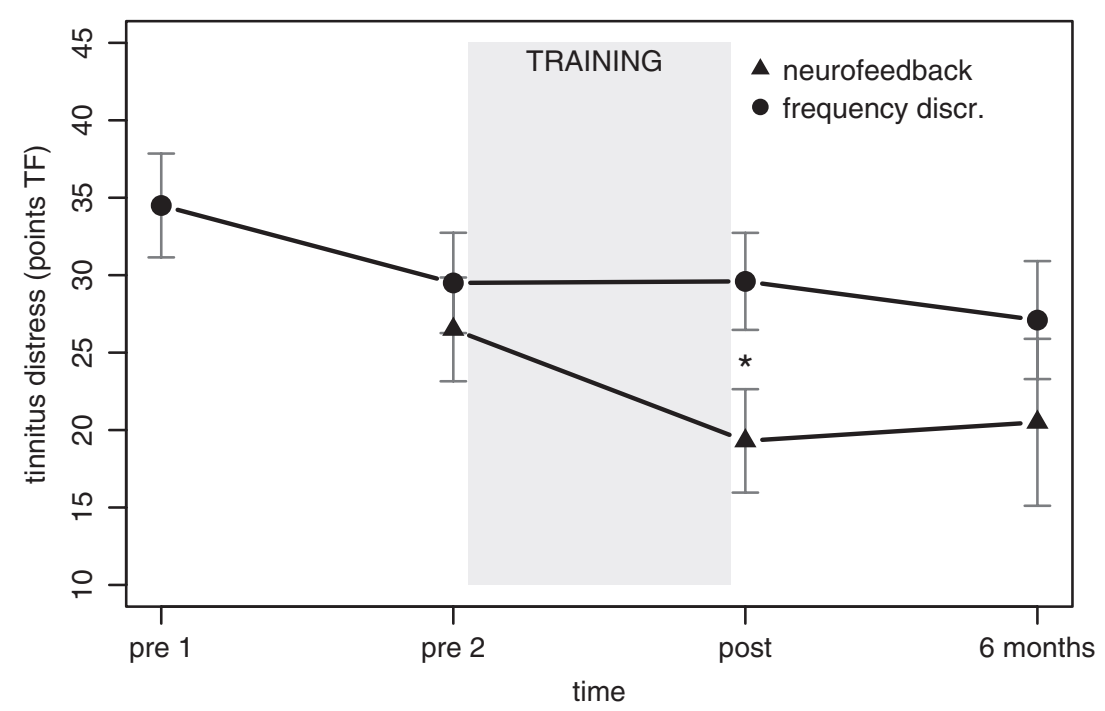

Fig. 4. Mean distress values $( \pm 1$ standard error) for the frequency discrimination group (triangles) at the initial interview (pre 1$)$, 1 week before training onset (pre 2), immediately following the training period (post), and 6 months follow-up, and the neurofeedback group (circles) before training (pre 2), after (post), and 6 months later (follow-up); values for "pre 1" are nonexistent. The gray bar displays the training period. The asterisk indicates a significant difference between the groups after the training period, whereas there is no significant difference at pre 2 and 6 months follow-up between the two training groups. Tinnitus distress was measured with the German Tinnitus Questionnaire (TF) and comprises values from 0 to 84 points, with high values indicating severe distress. Both samples have slight to moderate initial distress values $(0-46)$. 
training. Expectancy of treatment produced a reduction from 34.5 to 29.5 points on the Tinnitus Questionnaire before the actual start of the training (pre 1 to pre 2), but no further reduction in distress then occurred due to the training. The finding that the FDT had little success in alleviating distress in tinnitus is in agreement with the results of other studies (Flor et al., 2004).

\section{Relation between EEG normalization and tinnitus relief}

The participants who successfully modified their oscillatory brain activity had the greatest reduction in their tinnitus (Fig. 5) with a correlation $r=-.74(t(18)=-4.69, p<0.001)$. The correlations of change in the tau activity with the intensity of the tinnitus $(r=-0.29)$, and change in delta activity with tinnitus intensity $(r=0.37)$, were not statistically significant $(p>0.05)$. Concerning the distress, there is neither a significant correlation between the change of TDR and distress change $(r=0.22$, n.s. $)$, nor between each single frequency band with the distress change.

Changes in the TDR may reflect changes in tau, delta, or both. To determine the relevant predictive parameter(s), participants were classified in four groups based on their ability to modify tau and delta irrespective of the feedback protocol. Participants who modified both bands simultaneously had the strongest tinnitus relief and some even experienced complete relief from their tinnitus. The average reduction in tinnitus intensity was $71 \%$, which was significantly greater than achieved by the other three groups (Fig. 6; comparison with the "no change" group: $t=2.72, p=0.017$ ). Participants who only changed one band did not reduce their tinnitus significantly (tau: $26 \%$;

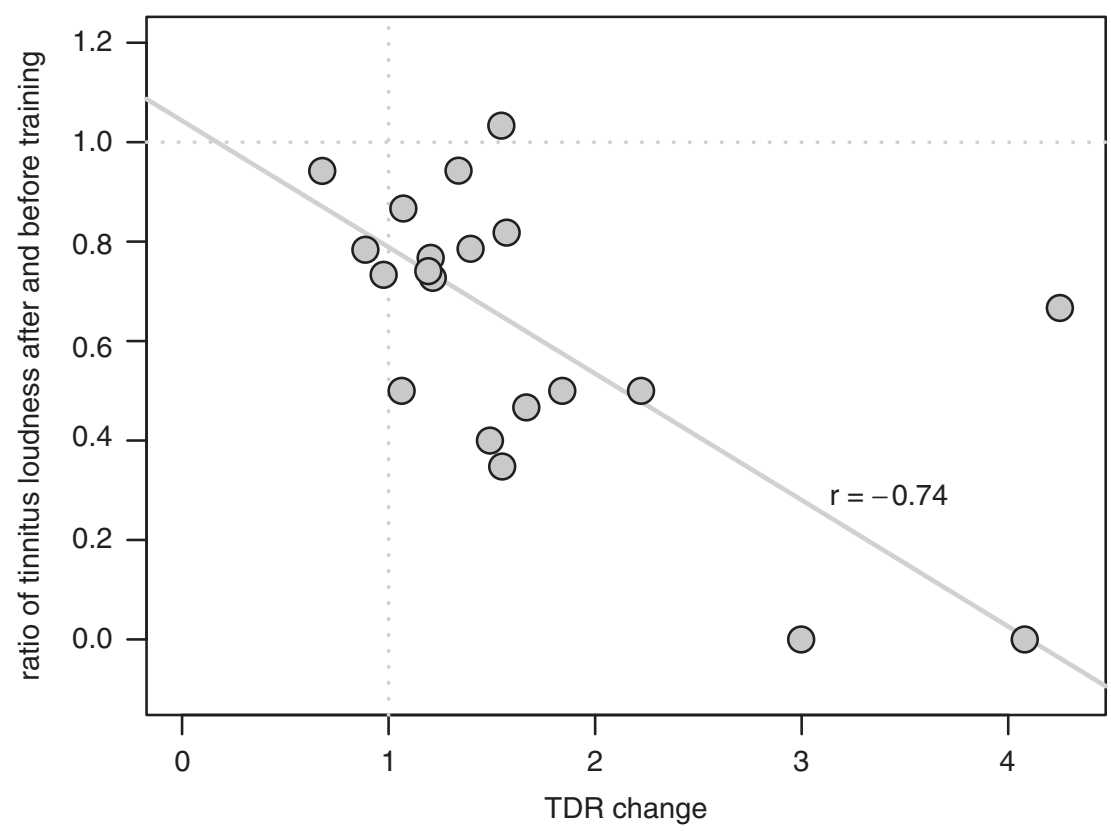

Fig. 5. Correlation between the change in tau/delta power ( $x$-axis; displayed as the tau/delta ratio after the training divided by the tau/ delta ratio before the training) and the change in tinnitus intensity ( $y$-axis; ratio between the intensity after the therapy and the intensity before the therapy). Values in tau/delta change above 1 (dashed line) indicate a high normalization ( = enhancement of tau and/or reduction of delta), whereas slight values in tinnitus intensity reduction (under the dashed line) indicate large reduction. Two patients with large normalization show a tinnitus change of zero, indicating no tinnitus at post-training. The added line is the regression line with the regressor of tinnitus intensity reduction and the predictor of tau/delta change. These analyses are independent from the different feedback protocols. 


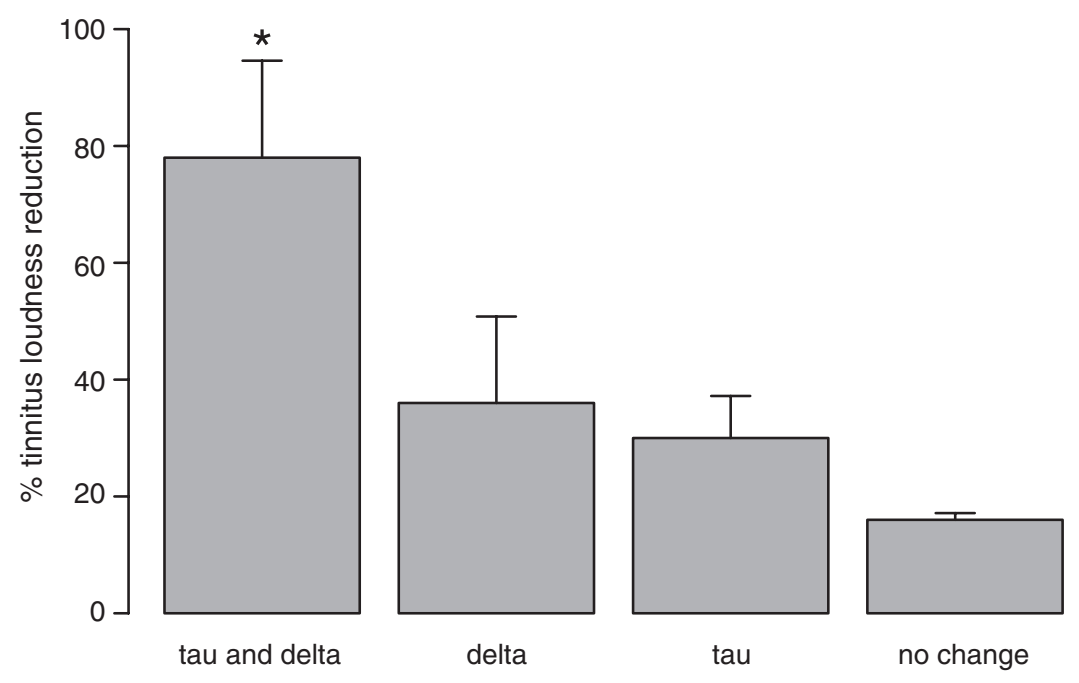

Fig. 6. Barplot of tinnitus intensity reduction depending on which frequency band could be modulated. This analysis is independent of the three different feedback protocols. The whole training group is divided into four clusters depending on the value of tau change prepost training and delta change pre-post training being above or under the median of changes. The left cluster comprises patients with values above the median of tau change and simultaneously under the median of delta change. These patients are referred to as the most successful ones as they were able to alter both bands in the desired direction. At the same time, they had the greatest tinnitus intensity reduction. The reduction is significantly higher than in subjects managing to alter delta (but not tau, second cluster from the left), or in the ones managing to change tau (but not delta, third cluster), and finally those subjects who neither enhanced tau nor reduced delta (fourth cluster). The reduction in the fourth cluster is $\sim 16 \%$, indicating a placebo effect.

delta: $36 \%$ reduction) compared to the patients who showed no change at all. The "no change" group also reduced their tinnitus intensity by $24 \%$, although these participants were not able to normalize tau or (nor?) delta, thereby representing an estimate of the placebo effects of treatment.

\section{Neurofeedback in the future: notions and suggestions for improvement}

The results described above indicate that neurofeedback may be an effective tool for treatment of chronic tinnitus patients. The neurofeedback protocol described above consisted of 10 training sessions dispersed across 4 weeks. Compared to other studies, this was a relatively small number of treatment days. Gosepath et al. (2001) treated their tinnitus participants for 12 days, Schenk et al. (2005) for 15 days. We conducted a pilot study with twice as many sessions (20 treatment days) over 4 weeks and TDR-feedback as usual. All four participants were able to normalize their power-spectrum during the entire therapy. A strong initial improvement was followed by a flattening of the learning curve during sessions 11-20 suggesting that longer trainings may bear additional but small benefits. The intensity of the training was greater in this group than the study described above with five trainings a week for four consecutive weeks. The competencies that patients learn during the therapy must be continued in their everyday life. We recommend that the use of neurofeedback therapy for chronic tinnitus include more than 10 sessions, $\sim 3$ days per week. Since the abnormal oscillations in chronic tinnitus sufferers have been occurring for several years, it requires a significant amount of exercise to return them to normal functioning.

\section{Instruction}

The results of the aforementioned neurofeedback study suggest that success (i.e., alleviation of 
tinnitus) depends on how much the individuals are able to normalize their brain rhythms (see Fig. 5). Participants who were not able to control their TDRs did not benefit from the training. This means that if this method is used in treatment of patients with teaching of the patients regarding how to gain control over their brain activity would likely improve the results of this method.

The original basis for neurofeedback is learning through operant conditioning (Travis et al., 1974; Rockstroh et al., 1984). Alternatively, in a study of neurofeedback the signal alone may be sufficient for training the participant. The feedback provided may raise awareness in the participant enough that self-regulation of brain activity can occur (Rockstroh et al., 1984). Therefore, participants do not learn from the therapist; rather they are provided feedback when a particular emotional or mental state is achieved and they learn through this feedback. The optimal conditions for improvement may indeed be a combination of a therapist or instructor teaching participants a behavior that can lead to normalization of brain rhythms and provide the participant with feedback. Currently, the workgroup of Del Bo (Milano, Italy) is investigating the effect of a combined tau/delta neurofeedback training, as proposed here, along with the Tinnitus Retraining Therapy (Jastreboff, 2006).

\section{Feedback protocol and electrode set-up}

We used three different feedback protocols in the study described above (TDR, tau alone, and delta alone). Regardless of the protocol those participants who were able to change the TDR in the desired direction benefited most from the training (see Fig. 6)

On the basis of these results, we concluded that the tau- and delta activity are not independent of each other but are systematically connected. This should be considered when this method is adapted for clinical use in treating patients with tinnitus. The ratio between the powers in these two EEG bands is not an optimal measure of success in changing the oscillations in the EEG for tinnitus suppression because changes in one band are enough to produce success in the training. We therefore currently investigate the effects of a protocol that enables us to provide specific feedback of the tau- and the delta band simultaneously. For that purpose we display delta power on the abscissa and tau power on the ordinate of the computer display used by the person who is undergoing treatment. A feedback symbol moves across the monitor in two-dimensional space. The person's task is to bring the symbol into the quadrant that represents an enhancement of tau- and a reduction of delta activity and make it stay there.

There are many possibilities as to where to place the electrodes for recording the EEG signal that is used for the training. In the present study we used four electrodes placed at $\mathrm{C} 3, \mathrm{C} 4, \mathrm{Fc} 1$, and $\mathrm{Fc} 2$. This setup was chosen because fronto-central electrodes are likely to pick up activity from the auditory cortex, which are largely tangentially oriented. However, the measured activity also represented frontal regions. To see whether training success is related to changes in temporal or in frontal sources and to investigate their relationship to the alleviation of tinnitus symptoms, whole-head EEG-data might be helpful and are also currently implemented in our ongoing neurofeedback study.

\section{Prediction of training success}

It would be beneficial to be able to predict how successful the training of a specific person would be and that would make it possible to identify patients who would benefit most from this form of neurofeedback therapy. For that purpose we performed a post-hoc regression analysis with the pre/post-reduction of tinnitus loudness as the dependent variable and with the following regressors: pre tau power, pre delta power, pre/postchange of the TDR, pre tinnitus loudness (dB), pre tinnitus severity (measured with the "Tinnitus Questionnaire"), duration of tinnitus, age, sex, 
and side of tinnitus perception. The power of tau and delta before therapy, as well as the normalization of both frequency bands (TDR pre/post), were the only significant predictors for the reduction of tinnitus loudness. The model indicates that parameters of the individual tinnitus perception such as the duration of tinnitus, side of perception, severity and loudness of tinnitus, or subject-specific factors such as age and sex, do not influence the outcome of the therapy. It is rather the pattern of oscillatory activity before and the amount of normalization during the therapy that determines the success in reducing the loudness of tinnitus. The prediction of the model was highly significant $\left(p=0.001, F_{9,10}=10.1\right)$ and explained $81 \%$ of the variance in tinnitus loudness reduction (adjusted $R^{2}$ of 0.811 ).

In addition, we found the duration of tinnitus to be negatively related to the success of the treatment $(r=-.41, p=0.08)$. Thus, patients with a short history of tinnitus profit most from the neurofeedback training. Given the assumption that there is a network of ongoing pathological neural oscillations producing tinnitus, this observation seems plausible. As a result, the longer the history of tinnitus, the longer the history of abnormal oscillations. These oscillations may then form a stabilized neural network over time. If a patient begins neurofeedback training shortly after the onset of tinnitus onset, the connections of the tinnitus network may not yet be consolidated and are thus more likely to revert back to a more regular activity level.

\section{Summary}

The use of neurofeedback in the treatment of chronic tinnitus is an emerging field of research. Our approach is based on the modulation of specific abnormal oscillations. We focus on the normalization of the tau-to-delta power ratio, generated in temporal and possibly frontal areas. The greatest reduction in the loudness of tinnitus occurred in participants who were capable of normalizing the TDR. Participants in this study benefited more than participants in a study using auditory discrimination training to reduce tinnitus. Modulation of specific abnormal brain oscillations via neurofeedback seems to be a potential route for alleviating the intensity and related psychological distress of chronic tinnitus.
Abbreviations
$\mathrm{AD} / \mathrm{HD}$ attention deficit/hyperactivity disorders
EEG electroencephalography
FDT frequency discrimination training
MEG magnetoencephalography
TDR tau-to-delta ratio

\section{Acknowledgments}

Our research is funded by the Deutsche Forschungsgemeinschaft and the Tinnitus Research Initiative. We would like to thank Isabel Lorenz, Anke Trefz, Susanne Völk, and Sylvia Datson for support during data collection.

\section{References}

Andersson, G. and Lyttkens, L. (1999) A meta-analytic review of psychological treatments for tinnitus. Br. J. Audiol., 33: 201-210.

Andersson, G., Stromgren, T., Strom, L. and Lyttkens, L. (2002) Randomized controlled trial of internet-based cognitive behavior therapy for distress associated with tinnitus. Psychosom. Med., 64: 810-816.

Dobie, R.A. (1999) A review of randomized clinical trials in tinnitus. Laryngoscope, 109(8): 1202-1211.

Dohrmann, K., Elbert, T.R., Schlee, W. and Weisz, N. (2007) Tuning the tinnitus percept by modification of synchronous brain activity. Restor. Neurol. Neurosci. Submitted.

Duffy, F.H. (2000) The state of EEG biofeedback therapy (EEG operant conditioning) in 2000: in an editor's opinion. Clin. Electroencephalogr., 31: 5-7.

Eggermont, J.J. and Roberts, L.E. (2004) The neuroscience of tinnitus. Trends Neurosci., 27: 676-682.

Elbert, T. and Heim, S. (2001) A light and a dark side. Nature, 411: 139.

Elbert, T., Rockstroh, B., Canavan, A., Birbaumer, N., Lutzenberger, W. and von Bülow, I. (1991) Self-regulation of slow cortical potentials and its role in epilepto genesis. 
In: Seifert J.G.C.R. (Ed.), International Perspectives on Self-Regulation and Health. Plenum Press, New York, pp. 65-94.

Flor, H., Elbert, T., Knecht, S., Wienbruch, C., Pantev, C., Birbaumer, N., Larbig, W. and Taub, E. (1995) Phantom-limb pain as a perceptual correlate of cortical reorganization following arm amputation. Nature, 375: 482-484.

Flor, H., Hoffmann, D., Struve, M. and Diesch, E. (2004) Auditory discrimination training for the treatment of tinnitus. Appl. Psychophysiol. Biofeedback, 29: 113-120.

Goebel, G. (2001) Wirksamkeit psychotherapeutischer verfahren. In: Goebel G. (Ed.), Ohrgeräusche: Psychosomatische Aspekte des Komplexen Chronischen Tinnitus. Urban \& Vogel, München, pp. 97-123.

Goebel, G. and Hiller, W. (1998) Tinnitus-Fragebogen (TF): Ein Instrument zur Erfassung von Belastung und Schweregrad bei Tinnitus. Hogrefe, Göttingen.

Gosepath, K., Nafe, B., Ziegler, E. and Mann, W.J. (2001) Neurofeedback in therapy of tinnitus. HNO, 49: 29-35.

Hari, R. and Salmelin, R. (1997) Human cortical oscillations: a neuromagnetic view through the skull. Trends Neurosci., 20: 44-49.

Henry, J. and Meikle, M. (2000) Psychoacoustic measures of tinnitus. J. Am. Acad. Audiol., 11: 138-155.

Hensel, S., Rockstroh, B., Berg, P., Elbert, T. and Schonle, P.W. (2004) Left-hemispheric abnormal EEG activity in relation to impairment and recovery in aphasic patients. Psychophysiology, 41: 394-400.

Jastreboff, P.J. and Jastreboff, M.M. (2006) Tinnitus retraining therapy: a different view on tinnitus. ORL J. Otorhinolaryngol. Relat. Spec., 68: 23-29 Discussion 29-30.

Jeanmonod, D., Magnin, M. and Morel, A. (1996) Low-threshold calcium spike bursts in the human thalamus: common physiopathology for sensory, motor and limbic positive symptoms. Brain, 119(Pt 2): 363-375.

de Jongh, A., Baayen, J.C., de Munck, J.C., Heethaar, R.M., Vandertop, W.P. and Stam, C.J. (2003) The influence of brain tumor treatment on pathological delta activity in MEG. NeuroImage, 20: 2291-2301.

Kalcioglu, M.T., Bayindir, T., Erdem, T. and Ozturan, O. (2005) Objective evaluation of the effects of intravenous lidocaine on tinnitus. Hear. Res., 199: 81-88.

Landis, B. and Landis, E. (1992) Is biofeedback effective for chronic tinnitus? An intensive study with seven subjects. Am. J. Otolaryngol., 13: 349-356.

Langguth, B., Hajak, G., Kleinjung, T., Pridmore, S., Sand, P. and Eichhammer, P. (2006) Repetitive transcranial magnetic stimulation and chronic tinnitus. Acta. Otolaryngol. Suppl.: 102-104.

Lehtela, L., Salmelin, R. and Hari, R. (1997) Evidence for reactive magnetic $10 \mathrm{~Hz}$ rhythm in the human auditory cortex. Neurosci. Lett., 222: 111-114.

Llinas, R., Urbano, F.J., Leznik, E., Ramirez, R.R. and van Marle, H.J. (2005) Rhythmic and dysrhythmic thalamocortical dynamics: GABA systems and the edge effect. Trends Neurosci., 28: 325-333.

Muhlnickel, W., Elbert, T., Taub, E. and Flor, H. (1998) Reorganization of auditory cortex in tinnitus. Proc. Natl. Acad. Sci. U.S.A., 95: 10340-10343.

Norena, A. and Eggermont, J. (2003) Changes in spontaneous neural activity immediately after an acoustic trauma: implications for neural correlates of tinnitus. Hear. Res., 183: 137-153.

Nowlis, D.P. and Kamiya, J. (1970) The control of electroencephalographic alpha rhythms through auditory feedback and the associated mental activity. Psychophysiology, 6: 476-484.

Ramirez, P.M., Desantis, D. and Opler, L.A. (2001) EEG biofeedback treatment of ADD: a viable alternative to traditional medical intervention. Ann. N. Y. Acad. Sci., 931: 342-358.

Rief, W., Weise, C., Kley, N. and Martin, A. (2005) Psychophysiologic treatment of chronic tinnitus: a randomized clinical trial. Psychosom. Med., 67: 833-838.

Rockstroh, B., Birbaumer, N., Elbert, T. and Lutzenberger, W. (1984) Operant control of EEG and event-related and slow brain potentials. Biofeedback Self-Regul., 9(2): 139-160.

Rockstroh, B., Elbert, T., Birbaumer, N., Wolf, P., DuchtingRoth, A., Reker, M., Daum, I., Lutzenberger, W. and Dichgans, J. (1993) Cortical self-regulation in patients with epilepsies. Epilepsy Res., 14: 63-72.

Rockstroh, B., Elbert, T., Lutzenberger, W. and Birbaumer, N. (1990) Evaluation and therapy in children with attentional dysfunctions. In: Rothenberger A. (Ed.), Brain and Behavior in Child Psychiatry. Springer, Berlin, pp. 345-357.

Sauseng, P., Klimesch, W., Doppelmayr, M., Pecherstorfer, T., Freunberger, R. and Hanslmayr, S. (2005) EEG alpha synchronization and functional coupling during top-down processing in a working memory task. Hum. Brain Mapp., 26: $148-155$.

Schenk, S., Lamm, K., Gundel, H. and Ladwig, K.H. (2005) Effects of neurofeedback-based EEG alpha and EEG beta training in patients with chronically decompensated tinnitus. HNO, 53: 29-37.

Steriade, M. and Timofeev, I. (2003) Neuronal plasticity in thalamocortical networks during sleep and waking oscillations. Neuron, 37: 563-576.

Strik, C., Klose, U., Kiefer, C. and Grodd, W. (2002) Slow rhythmic oscillations in intracranial CSF and blood flow: registered by MRI. Acta Neurochir. Suppl., 81: 139-142.

Tanaka, A., Kimura, M., Yoshinaga, S., Tomonaga, M. and Mizoguchi, T. (1998) Quantitative electroencephalographic correlates of cerebral blood flow in patients with chronic subdural hematomas. Surg. Neurol., 50: 235-240.

Travis, T.A., Kondo, C.Y. and Knott, J.R. (1974) Alpha conditioning: a controlled study. J. Nerv. Ment. Dis., 158: 163-173. 
Walter, G. (1936) The location of cerebral tumours by electroencephalography. Lancet, 8: 305-308.

Weber, C., Arck, P., Mazurek, B. and Klapp, B.F. (2002) Impact of a relaxation training on psychometric and immunologic parameters in tinnitus sufferers. J. Psychosom. Res., 52: 29-33.

Weisz, N., Dohrmann, K., and Elbert, T. (2007) The relevance of sportaneous activity for the coding of the tinnitus sensation. In press.
Weisz, N., Hartmann, T., Dohrmann, K., Schlee, W. and Norena, A., (2006) High-frequency tinnitus without hearing loss does not mean absence of deafferentation, Hear. Res. doi:10.1016/j.heares.2006.09.003.

Weisz, N., Moratti, S., Meinzer, M., Dohrmann, K. and Elbert, T. (2005) Tinnitus perception and distress is related to abnormal spontaneous brain activity as measured by magnetoencephalography. PLoS Med., 2: e153. 

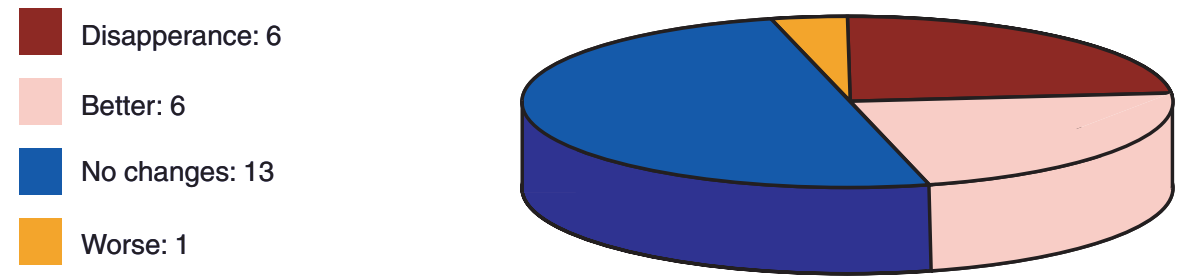

Plate 37.2. Response to TENS treatment after 2 weeks. Tinnitus improvement was referred by $46 \%$ of the patients (disappearance + better). (For B/W version, see page 392 in the volume.)

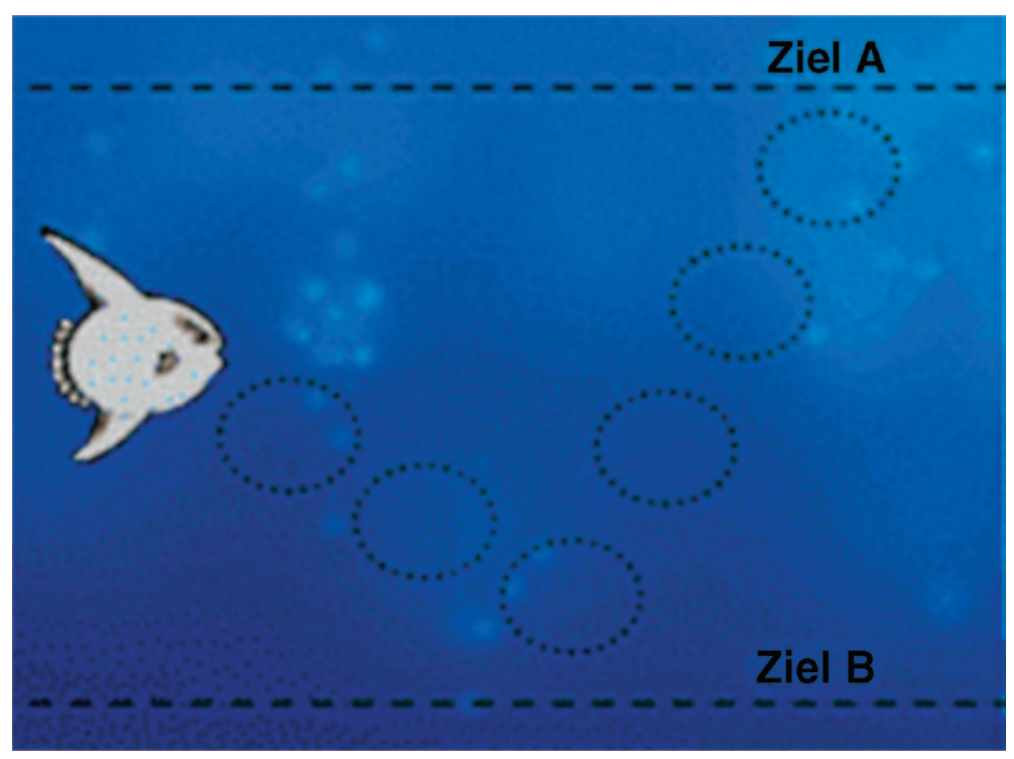

Plate 46.2. The schematic fish on the patient monitor that was to be "moved" up or down (depending on the feedback protocol) during neurofeedback training. The height of the fish represented the amplitude/power of the specific frequency band. (By courtesy of Eldith $\mathbb{C}$ Corporation, Germany.) (For B/W version, see page 477 in the volume.) 